\title{
Microbial keratitis in corneal grafts: predisposing factors and outcomes
}

ACO Okonkwo ${ }^{1,2}$, WF Siah ${ }^{1}$, HDJ Hogg ${ }^{1,2}$, $\mathrm{H}$ Anwar ${ }^{1}$ and FC Figueiredo ${ }^{1,2}$

\begin{abstract}
Purpose To identify the nature of microbial keratitis in corneal grafts and the clinical outcomes at a tertiary hospital in the United Kingdom.

Patients and methods A retrospective case series of microbial keratitis in corneal grafts at the Royal Victoria Infirmary, Newcastle upon Tyne over a 17-year period (1997-2014). Results A total of 759 consecutive corneal grafts were identified from the Cornea Transplantation database. Of these, 59 episodes of microbial keratitis occurred in 41 eyes of 41 patients $(5.4 \%$; 19 male, $46.3 \%$ ). Median patient age was 73 years ( $\mathrm{SD}=19.4$ years). The most common indication for corneal transplantation was bullous keratopathy (11/41, 26.8\%). There were $34 / 59(57.6 \%)$ episodes of culture-positive graft keratitis; Streptococcus pneumoniae and Staphylococcus aureus were each isolated in $5 / 34(14.7 \%)$ culturepositive episodes. In all, $35 / 59(59.3 \%)$ episodes of microbial keratitis occurred in 22 previously failed grafts and 3 de novo graft failures. Gram-negative keratitis was more likely to cause reduced BCVA after $\left(\chi^{2}\right.$-test, $\left.P=0.02\right)$. Median graft duration was 49.5 months ( $\mathrm{SD}=43.7$ months). Failed grafts were significantly older (median 69 vs 27 months, $P=0.009$ ).

Conclusion This represents the longest published follow-up data on microbial keratitis and is the only of its kind in the United Kingdom. The incidence of $5.4 \%$ is comparable to that within the developed world. Graft age was significantly associated with graft failure in microbial keratitis; the ongoing risk of microbial keratitis warrants providing patients with long-term open access to hospital eye services. Eye (2018) 32, 775-781; doi:10.1038/eye.2017.310; published online 26 January 2018
\end{abstract}

\section{Introduction}

A total of 3779 keratoplasties were performed in the United Kingdom between April 2014 and April 2015. ${ }^{1}$ Common indications for keratoplasty include bullous keratopathy and Fuch's endothelial dystrophy. ${ }^{2-4}$ Microbial keratitis is a major complication of keratoplasty that poses serious ocular morbidities, including reduced best-corrected visual acuity (BCVA) and graft failure. Therefore, early diagnosis, prompt treatment, and close surveillance are required to reduce patient morbidity. ${ }^{2}$ The incidence of microbial keratitis in grafts varies from 1.8-7.4\% in the developed world to $11.9-25 \%$ in the developing world. ${ }^{2,5-13}$ Predisposing factors are thought to include (but not be limited to); ocular surface diseases such as keratoconjunctivitis sicca, atopy, previous herpes simplex keratitis; patient age; graft duration; suture-related problems; and pre-keratitis graft failure. ${ }^{11-17}$ (Figure 1).

Up to $77 \%$ of culture-positive microbial graft keratitis are caused by Gram-positive pathogens. The most commonly implicated Gram-positive pathogens are Staphylococcus aureus and Streptococcus pneumoniae whilst the most frequently isolated Gram-negative species are Pseudomonas aeruginosa and Moraxella. $2,6,6,9,9,11-14,17,18$ However, isolation rates have been reported to be only $33 \%$ as patients presenting with microbial graft keratitis have often been treated with antimicrobials before culture specimens are obtained. ${ }^{19}$

This retrospective study aims to identify the incidence, nature, and outcomes of microbial keratitis occurring in all types of corneal grafts at the Royal Victoria Infirmary hospital, Newcastle upon Tyne, a tertiary corneal transplantation referral centre in the United Kingdom.

Furthermore, this study aims to retrospectively identify predisposing factors for microbial keratitis in corneal grafts and their subsequent impact on clinical outcomes.
${ }^{1}$ Newcastle University, Newcastle upon Tyne, UK

${ }^{2}$ Department of Ophthalmology, Royal Victoria Infirmary, Newcastle upon Tyne, UK

Correspondence: ACO Okonkwo, Hull Royal Infirmary, Analby Road, Hull, UK

Tel: +07 999121893 ; Fax: +014 82875875 E-mail: Arthur.okonkwo@ doctors.org.uk

Received: 3 February 2017 Accepted in revised form: 18 November 2017 Published online: 26 January 2018 


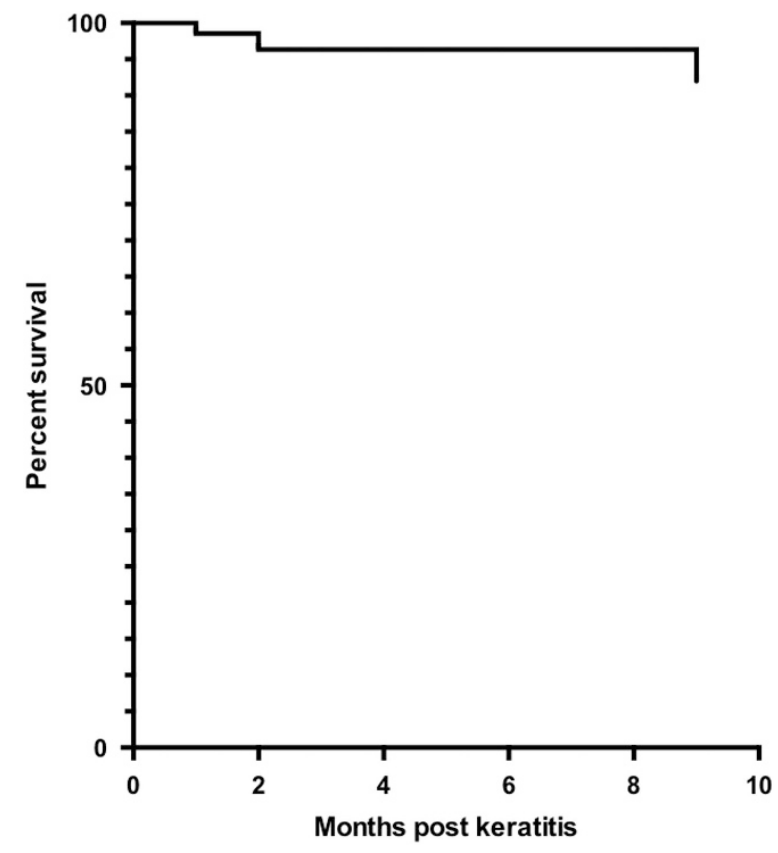

Figure 1 Kaplan-Meier plot showing graft success after microbial keratitis in viable grafts ( $n=24$ episodes).

\section{Materials and Methods}

A retrospective review of the Cornea Transplantation Service database at a tertiary referral Ophthalmology Department (Royal Victoria Infirmary, Newcastle upon Tyne, UK) was undertaken to identify patients who were treated for microbial keratitis in corneal grafts between January 1997 and July 2014. All keratoplasties were either carried out by the senior author (FCF) or under his direct supervision. In all penetrating keratoplasties (PKPs), the donor graft buttons were oversized by $0.25 \mathrm{~mm}$ in diameter and sutured with 10/0 interrupted sutures (Ethicon, Livingston, UK) and 11/0 nylon continuous suture (Ethicon), respectively. Deep anterior lamellar keratoplasties (DALKs) were performed using previously reported Melles Technique with minor modifications whilst Descemet's stripping automated endothelial keratoplasties (DSAEKs) were performed using the technique reported by Müller et al. ${ }^{20,21}$ Selective removal of 10/0 sutures starts 8 weeks postoperatively guided by refraction and topography. Subsequently sutures are removed at 8-10 week intervals. When corneal astigmatism reaches $<3 \mathrm{D}$ sutures are only removed if they break or are loose. 11-0 sutures stay indefinitely unless they break or are loose.

Patients receive prophylactic topical antibiotics for 4 weeks in the immediate postoperative period and for 1 week after suture removal. Patients are routinely followed up for the length of their graft unless they fail to attend, transfer to another centre, or die.
Episodes of bacterial and fungal keratitis were included in the study whilst any case of herpetic keratitis was excluded. A retrospective analysis of all identified consecutive patients' medical records was performed. Data collection included patient demographics, predisposing factors, clinical and microbiological findings, and final outcomes (change in BCVA, graft failure, and/or graft rejection). The tenets of the Declaration of Helsinki were adhered to. The Newcastle upon Tyne Hospitals' Trust Clinical Governance Department had approved this study for prospective data collection and retrospective audit of corneal transplantation surgery outcomes, respectively.

Statistical analysis was performed using Prism version 6.00 for Mac, GraphPad Software, La Jolla, CA, USA. To assess for data normality, Shapiro-Wilk test was used when the sample size was $n \leq 50$, and KolmogorovSmirnov test when $n>50$. $\chi^{2}$-test and Mann-Whitney $U$ test were used for categorical and nonparametric data, respectively. Student's $t$-test was used to compare differences in two parametric groups. A $P$-value of $<0.05$ was considered statistically significant.

The data collected in the RVI PKP registry is prospective with some additional information collected from the medical notes as required for evaluation of the corneal transplantation service.

\section{Results}

A total of 759 consecutive corneal grafts were performed on 558 eyes (including 201 regrafts on 201 eyes); 685 (90.3\%) PKPs; 20 (2.6\%) DALKs; and 54 (7.1\%) DSAEKs. In all, 220 patients were lost follow-up, transferred to another centre, or died.

Fifty-nine episodes of microbial keratitis were identified in 41 eyes of 41 patients (39 PKPs and 2 DALKs). The most common indication for corneal transplantation in these eyes was for aphakic/pseudophakic bullous keratopathy ( $n=11 / 41,26.8 \%$; Table 1). In all, 30/41 (73.2\%) developed a single episode of microbial keratitis (30 PKP) whilst $11 / 41(26.8 \%)$ patients with microbial keratitis developed multiple (2 or more) episodes of microbial keratitis in the same eye (9 PKPs and 2 DALKs). The overall incidence of microbial keratitis in corneal grafts was $41 / 759,5.4 \%$ (39/685, 5.7\% in PKP; 2/20, 10\% in DALK; and $0 / 54,0 \%$ in DSAEK). The incidence of multiple episodes of microbial keratitis was $11 / 759,1.4 \%(26.8 \%$ as a proportion of episodes of microbial keratitis). Overall, 35/59 (59.3\%) episodes of microbial keratitis in 22 eyes occurred in failed grafts. There were $3 / 24(12.5 \%)$ de novo graft failures post keratitis in the remaining 24 episodes of microbial keratitis in 19 viable grafts (Figure 2). A total of 15/59 (25.4\%) episodes of microbial keratitis resulted in a persistent epithelial defect (PED) at 1 month from diagnosis of 
Table 1 Indication for keratoplasty

\begin{tabular}{lcc}
\hline Indication & All grafts, $\mathrm{N}=41$ & $\begin{array}{c}\text { Number of grafts } \\
\text { experiencing multiple episodes } \\
\text { of microbial keratitis, } \mathrm{N}=11\end{array}$ \\
\hline Aphakic/pseudophakic bullous keratopathy & 11 & 6 \\
Fuch's endothelial dystrophy & 8 & 1 \\
Herpes simplex scar & 5 & 2 \\
Failed graft & 4 & 0 \\
Perforated cornea & 3 & 0 \\
Keratoconus & 3 & 2 \\
Corneal scar & 3 & 0 \\
Peter's anomaly & 1 & 0 \\
Penetrating injury & 1 & 0 \\
Lattice corneal dystrophy & 1 & 0 \\
Interstitial keratitis & 1 & 0 \\
\hline
\end{tabular}

${ }^{a}$ Old herpes simplex scar without reactivation.

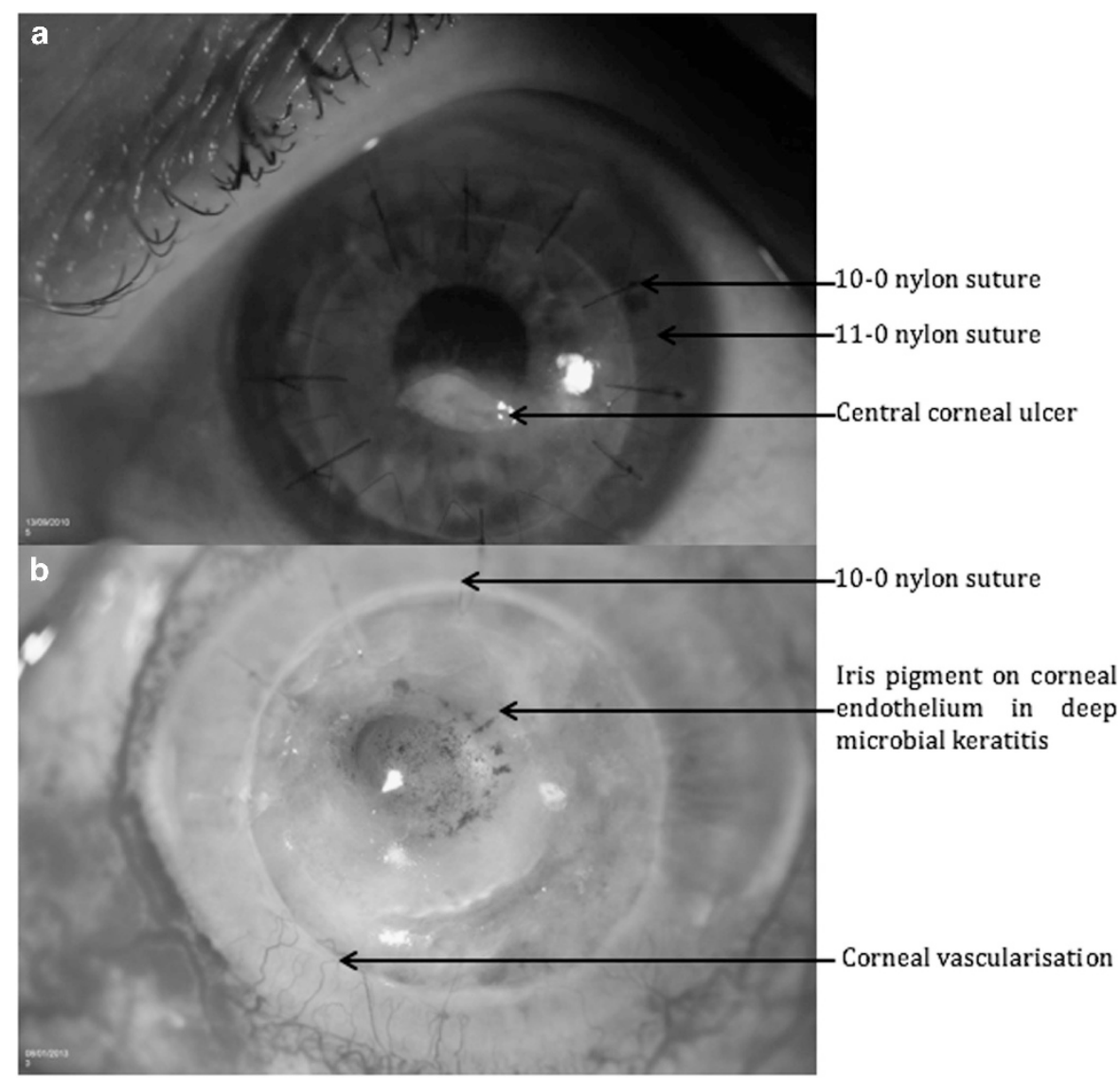

Figure 2 (a) Central corneal ulcer in viable graft. (b) Deep microbial keratitis in failed graft with corneal vascularisation.

microbial graft keratitis. A total of $9 / 15(60 \%)$ patients had clear grafts after a period of topical antimicrobial treatment with adjunctive bandage contact lens, 4/15 (26.7\%) developed corneal scarring, and 1/15 (6.7\%) subsequently developed a failed graft. One out of $15(6.7 \%)$ patients had been transferred to a peripheral unit and the clinical outcome was unknown.
Visual outcome data were available for 58/59 episodes of microbial keratitis in $40 / 41$ grafts. One-third of the episodes of microbial keratitis $(19 / 58,32.8 \%)$ resulted in reduced BCVA post keratitis with a median loss of 2 Snellen lines (range 1-7). In all, $46 / 58$ episodes (78.0\%) of microbial keratitis required hospitalisation with a median stay of 7 days $(S D=6.2$ days $)$. 


\section{Demographics}

A total of 21/41 (51.2\%) patients were male. Median age of patients was 73 years ( $S D=19.4$ years). Patient age was not a significant factor for poor clinical outcomes such as reduced BCVA (median 75 vs 71.6 years, $P>0.9$ ) and graft failure (median 76.8 vs 70 years, $P=0.3$ ). Median graft duration was 49.5 months ( $\mathrm{SD}=43.7$ months). Failed corneal grafts were older than viable grafts (mean 74.7 (SD=41.4) vs 44.9 (SD=41.7), $P=0.01)$. Grafts with reduced $\mathrm{BCVA}$ post keratitis were not older than grafts without reduced BCVA post keratitis (mean 53.2 ( $\mathrm{SD}=43.3$ months) vs 56.9 ( $\mathrm{SD}=44.3$ months), $P=0.8)$.

\section{Predisposing factors}

Predisposing factors for microbial keratitis were documented in 57/59 episodes of microbial keratitis. Premorbid failed graft $(35 / 57,61.4 \%)$ and concurrent topical glaucoma treatment $(34 / 57,59.6 \%)$ were commonly observed. Suture-related microbial keratitis was found in $19.3 \%(11 / 57)$ of cases. Multiple potential predisposing factors were evident in 44/57 episodes of microbial keratitis, a single predisposing factor in 12/57 episodes, and no obvious predisposing factor in $1 / 57$ episodes (median 2). 54/57 (94.7\%) episodes of keratitis occurred in grafts taking topical steroids (frequency and type of steroid was not collected).

\section{Microbiology}

Microbiology results were available for all 59 episodes. In all, 25/59 (42.4\%) corneal scrapes reported no growth, 18/59 (30.5\%) grew Gram-positive organisms, 11/59 (18.6\%) Gram-negative organisms, and 5/59 (8.5\%) fungi. Prevalent organisms isolated were $S$. pneumoniae and $S$. aureus each was isolated in five episodes and $P$. aeruginosa, which was isolated in four episodes (Table 2).

Those who had Gram-positive and Gram-negative keratitis did not significantly vary in age (Mann-Whitney $U$-test $P=0.3$ ) or age of graft (Mann-Whitney $U$-test, median 53.5 vs 50.5 years, $P=0.8$ ).

\section{Post-microbial keratitis outcomes}

Post-microbial keratitis outcomes were unavailable for 1 patient with Gram-negative keratitis. A total of 8/18 (44.4\%) episodes of Gram-positive keratitis occurred in failed grafts compared to 4/11 (40\%) episodes of Gramnegative keratitis and $0 / 5(0 \%)$ of fungal keratitis. There was no significant difference in organism type in those who suffered from graft failure and those who did not (Chi-Square test, $P=0.3) .3 / 18(16.7 \%)$ episodes of
Table 2 Microbiological profile of bacterial isolates from graft keratitis

\begin{tabular}{llr}
\hline Gram stain & Culture & $\mathrm{n}$ \\
\hline \multirow{2}{*}{ Gram-positive organism } & Staphylococcus aureus & 5 \\
& Streptococcus pneumoniae & 5 \\
& Staphylococcus epidermis & 2 \\
& Diphtheria & 1 \\
& Group G streptococcus & 1 \\
& Alpha Haemolytic streptococcus & 1 \\
& Streptococcus oralis & 1 \\
& Propionibacterium acnes & 1 \\
& Other & 1 \\
& Pseudomonas aeruginosa & 4 \\
Gram-negative organism & 2 \\
& Moraxella nonliquefaciens & 2 \\
& Achromobacter & 1 \\
& Haemophilus influenza & 1 \\
& Alcaligenes faecalis & 1 \\
\hline
\end{tabular}

gram-positive keratitis resulted in reduced BCVA (median 2 Snellen lines, $\mathrm{SD}=1)$ compared to $6 / 11(60 \%)$ episodes of Gram-negative keratitis (median 1, SD =0.5) and $0 / 5(0 \%)$ episodes of fungal keratitis. Those with Gram-negative keratitis were significantly more likely to have reduced final BCVA compared to those with Grampositive keratitis $\left(\chi^{2}\right.$-test, $\left.P=0.02\right)$.

\section{Corneal ulcers}

Corneal ulcer size Corneal ulcer size was recorded in $36 / 59$ episodes (61\%); 14/36 ulcers (38.9\%) were $<2 \mathrm{~mm}^{2}$ in size and $22 / 36$ ulcers $(61.1 \%)$ were $>2 \mathrm{~mm}^{2}$. Five out of $14(35.7 \%)$ grafts with ulcers $<2 \mathrm{~mm}^{2}$ and $11 / 22(50 \%)$ grafts with ulcers $>2 \mathrm{~mm}^{2}$ had post-keratitis graft failure. One out of $14(7.1 \%)$ graft with ulcer size $<2 \mathrm{~mm}^{2}$ and $8 / 22(36.4 \%)$ grafts with ulcers $>2 \mathrm{~mm}^{2}$ had reduced BCVA post keratitis. Larger ulcers were associated with worse BCVA $\left(\chi^{2}\right.$-test, $\left.P<0.05\right)$ but not graft failure $\left(\chi^{2}\right.$-test, $P=0.4)$.

There was no significant difference between the size of ulcer formed by different microorganisms (MannWhitney $U P=0.63$ ).

Corneal ulcer location Ulcer location was recorded in $38 / 59$ episodes and visual outcomes were available in $37 / 38$. Out of $38,25(65.8 \%)$ were located centrally, $12 / 38$ ulcers $(31.6 \%)$ at the graft-host junction, and 1/38 (2.6\%) was purely in the host region. Eight out of 25 (32\%) central ulcers resulted in worse BCVA compared to 2/12 $(16.7 \%)$ ulcers at the graft-host junction $\left(\chi^{2}\right.$-test, $\left.P=0.4\right)$. In all, $15 / 25(60 \%)$ central ulcers occurred in failed grafts failure, compared to $1 / 12(9.1 \%)$ at the graft-host junction $\left(\chi^{2}\right.$-test, $\left.P=0.003\right)$. 
Table 3 Complications per eye following 58 episodes of microbial keratitis in 41 eyes

\begin{tabular}{lcc}
\hline Complications & $\mathrm{n}$ & $\%$ \\
\hline Corneal scar & 16 & 39 \\
Persistent epithelial defect ( $>1$ month) & 16 & 39 \\
Corneal neovascularisation & 6 & 15 \\
Graft rejection & 3 & 7.3 \\
New graft failure & 3 & 7.3 \\
Perforation & 2 & 4.9 \\
\hline
\end{tabular}

\section{Complications}

Data on complications were available for 58/59 episodes in $41 / 41$ eyes. As stated previously, 3 eyes developed de novo graft failure after episodes of microbial keratitis. Other complications seen include corneal scarring, persistent epithelial defect, neovascularisation, graft rejection, and corneal perforation (Table 3).

Clinical outcomes and complications Four out of 14 (28.6\%) of those with ulcers $<2 \mathrm{~mm}^{2}$ developed a PED compared to $11 / 22(50 \%)$ of those with ulcers $>2 \mathrm{~mm}^{2}\left(\chi^{2}\right.$-test, $P=0.2)$. Eight out of $16(50 \%)$ of those with a PED suffered from reduced BCVA compared with 11/47 (23.4\%) grafts without PEDs $\left(\chi^{2}\right.$-test, $\left.P=0.2\right)$.

Two out of $12(16.7 \%)$ ulcers at the graft-host junction resulted in corneal perforation while no central ulcers resulted in corneal perforation $\left(\chi^{2}\right.$-test, $\left.P=0.03\right)$.

\section{Discussion}

This study reports the clinical outcomes of a large series of microbial keratitis with the longest follow-up from a single tertiary eye referral service in the UK. At the Royal Victoria Infirmary, all patients who have corneal grafts are given a telephone contact emergency card with a list of potential ocular symptoms and signs that are suggestive of serious corneal graft problems; ie, redness, sensitivity to light, visual impairment, and pain. Patients are encouraged to call $24 \mathrm{~h}$ a day, 7 days a week, 365 days a year, and are seen as an emergency for prompt diagnosis and management.

The rate of microbial graft keratitis, $5.4 \%$, provides the only such published statistic based on UK data at the time of writing and is comparable to the $1.8-7.6 \%$ previously reported in the developed world..$^{5-7}$ A limitation of the reported rate is that 220 patients were discharged/ transferred to another centre/lost to follow-up and therefore could potentially present elsewhere with late complications.

It was beyond the scope of this study to assess any causal relationship between previously reported risk factors and the incidence of microbial keratitis in corneal grafts, however, numerous risk factors were reported (eg, suture problems, keratoconjunctivitis sicca, atopic keratoconjunctivitis; eczema, herpes simplex, glaucoma treatment; and post-microbial keratitis graft failure). Advancing patient age has been previously reported as a risk factor for graft failure, microbial keratitis, and subsequent reduced BCVA. ${ }^{10}$ Although in Newcastle upon Tyne, we did not find increased patient age to be significantly associated with post-keratitis reduced BCVA and graft failure after microbial keratitis.

Patients with failed corneal grafts at presentation had significantly older corneal grafts than those without graft failure; 74.7 vs 44.9 months $(P=0.01)$. Mean graft duration was 55.7 months ( $\mathrm{SD}=43.7$ months) in this study, which is longer than that shown in other studies; median 17 months in a study by Das et al, ${ }^{14}$ median 24.4 months in a study by Wagoner et al, ${ }^{10}$ and mean 26.3 months in a study by Wright et al. ${ }^{13}$ The longer graft duration seen in this study may be due to the comparatively longer followup, 1997-2014, than the studies reported above, 19982005 , and may skew graft duration upward. ${ }^{10,13,14}$

Culture-positive rates in clinical keratitis of $57.6 \%$ are significantly higher than the $33 \%$ reported by Kaye et al. ${ }^{19}$ Consistent with much of the previous literature, most culture-positive keratitis was caused by Gram-positive organisms (52.9\%). S. aureus (14.7\%), S. pneumoniae $(14.7 \%)$, and $P$. aeruginosa $(10.5 \%)$ were the most commonly isolated species in this study. These organisms have all been previously identified as common causes of post-graft microbial keratitis. 2,6,8,9,11-14,17 Gram-negative keratitis has been previously shown to stimulate a greater inflammatory response on the ocular surface and in this study, we showed that those with Gram-negative keratitis had significantly worse visual outcomes, though we did not find any association with graft failure. ${ }^{22}$ Topical antibiotics used at presentation to treat acute post-graft microbial keratitis in the Ophthalmology Department at the Royal Victoria Infirmary in Newcastle tend to be broad spectrum. They offer both Gram-positive and Gram-negative cover such as frequent topical ofloxacin $0.3 \%$ and cefuroxime $1 \%$ eye drops, this may change based on response to initial treatment, culture results, and antibiotic sensitivity. Unfortunately, commonly resistant organisms in cultures was not collected.

Failed grafts are more likely to have central ulcers, however, ulcers found at the graft-host junction are more likely to cause corneal perforation than central ulcers. Two patients presented with corneal perforation: both of those were ulcers at the graft-host junction, one eye had underlying Peter's anomaly, failed graft, and Molteno tube; and one eye had a large staphylococcal ulcer in a failed graft. The patient with a Peter's anomaly underwent subsequent PKP and the patient with the staphylococcal ulcer now has an atrophic blind eye. 
The present study reports the longest follow-up of microbial keratitis in post-corneal grafts known to the authors at the time of writing. Reporting a single surgeon's experience gives a level of consistency to the study with little variation in a well-established corneal transplant service.

A limitation of this study was that records for a few episodes were not complete. Furthermore, this was a purely retrospective study investigating only grafts that experienced microbial keratitis and thus no comparison to grafts that did not suffer from microbial keratitis was made. In addition, this is a single-centre study and thus the cohort of patients may differ in different parts of the world and from different centres.

Finally, grafts may present elsewhere with late complications, potentially resulting in an underestimate of infection rate.

In summary, the incidence and nature of microbial post-graft keratitis in Newcastle is comparable to that within the developed world. The ongoing risk of microbial keratitis warrants giving patients with corneal grafts long-term open access to hospital eye services if they have any symptoms of microbial keratitis. Our study highlights that initial topical antibiotic therapy for suspected microbial keratitis should target Gram-positive organisms and Gram-negative Pseudomonas species. Clinicians should consider treating microbial keratitis more aggressively if it occurs in older grafts; additionally, although all ulcers are high risk those that are at the grafthost junction or $>2 \mathrm{~mm}^{2}$ in size should also be treated more aggressively to minimise morbidity.

\section{Summary}

What was known before

- Incidence of microbial keratitis in grafts is $1.8-7.4 \%$ in the developed world. No previous report of rates in the United Kingdom exists in the literature. Gram-positive pathogens cause $77 \%$ of culture-positive microbial graft keratitis. Predisposing factors to microbial graft keratitis include (but not be limited to) keratoconjunctivitis sicca, atopy, previous herpes simplex keratitis, patient age, graft duration, suture-related problems, and pre-keratitis graft failure.

\section{What this study adds}

- The rate microbial graft keratitis in the United Kingdom is $5.4 \%$, comparable to those previously reported in the developed world. The ongoing risk of microbial keratitis warrants giving patients access to emergency eye care in the event of acute eye problems. Initial topical antibiotic therapy for suspected microbial keratitis should target Gram-positive organisms and Pseudomonas species. Clinicians should treat microbial graft keratitis more aggressively if it occurs in older grafts; or if it affects the graft-host junction.

\section{Conflict of interest}

The authors declare no conflict of interest.

\section{References}

1 Transplant NBa. https://www.organdonation.nhs.uk/ supporting-my-decision/statistics-about-organ-donation/ transplant-activity-report/ Organ Donation and Transplantation Activity Report 2015/16. 2016.

2 Vajpayee RB, Sharma N, Sinha R, Agarwal T, Singhvi A. Infectious keratitis following keratoplasty. Surv Ophthalmol 2007; 52(1): 1-12.

3 Tixier J, Bourcier T, Borderie V, Laroche L. [Infectious keratitis after penetrating keratoplasty]. J Fr Ophtalmol 2001; 24(6): 597-602.

4 Tavakkoli H, Sugar J. Microbial keratitis following penetrating keratoplasty. Ophthalmic Surg 1994; 25(6): 356-360.

5 Harris DJ Jr., Stulting RD, Waring GO 3rd, Wilson LA. Late bacterial and fungal keratitis after corneal transplantation. Spectrum of pathogens, graft survival, and visual prognosis. Ophthalmology 1988; 95(10): 1450-1457.

6 Bates AK, Kirkness CM, Ficker LA, Steele AD, Rice NS. Microbial keratitis after penetrating keratoplasty. Eye (Lond) 1990; 4(Pt 1): 74-78.

7 Lamensdorf M, Wilson L, Waring G, Cavanagh H. Microbial keratitis after penetrating keratoplasty. Ophthalmology 1982; 89: 124.

8 Akova YA, Onat M, Koc F, Nurozler A, Duman S. Microbial keratitis following penetrating keratoplasty. Ophthalmic Surgery Lasers 1999; 30(6): 449-455.

9 Leahey AB, Avery RL, Gottsch JD, Mallette RA, Stark WJ. Suture abscesses after penetrating keratoplasty. Cornea 1993; 12(6): 489-492.

10 Wagoner MD, Al-Swailem SA, Sutphin JE, Zimmerman MB. Bacterial keratitis after penetrating keratoplasty: incidence, microbiological profile, graft survival, and visual outcome. Ophthalmology 2007; 114(6): 1073-1079.

11 Al-Hazzaa SA, Tabbara KF. Bacterial keratitis after penetrating keratoplasty. Ophthalmology 1988; 95(11): 1504-1508.

12 Moorthy S, Graue E, Jhanji V, Constantinou M, Vajpayee RB. Microbial keratitis after penetrating keratoplasty: impact of sutures. Am J Ophthalmol 2011; 152 (2):189-194.e2.

13 Wright TM, Afshari NA. Microbial keratitis following corneal transplantation. Am J Ophthalmol 2006; 142(6): 1061-1062.

14 Das S, Constantinou M, Ong T, Taylor HR. Microbial keratitis following corneal transplantation. Clin Exp Ophthalmol 2007; 35(5): 427-431.

15 Constantinou M, Jhanji V, Vajpayee RB. Clinical and microbiological profile of post-penetrating keratoplasty infectious keratitis in failed and clear grafts. Am J Ophthalmol 2013; 155(2): 233-237.e2.

16 Vajpayee RB, Boral SK, Dada T, Murthy GV, Pandey RM, Satpathy G. Risk factors for graft infection in India: a casecontrol study. Br J Ophthalmol 2002; 86(3): 261-265.

17 Tseng SH, Ling KC. Late microbial keratitis after corneal transplantation. Cornea 1995; 14(6): 591-594.

18 Sonavane A, Sharma S, Gangopadhyay N, Bansal AK. Clinico-microbiological correlation of suture-related graft infection following penetrating keratoplasty. Am J Ophthalmol 2003; 135(1): 89-91. 
19 Kaye SB, Rao PG, Smith G, Scott JA, Hoyles S, Morton CE et al. Simplifying collection of corneal specimens in cases of suspected bacterial keratitis. J Clin Microbiol 2003; 41(7): 3192-3197.

20 Melles G, Lander F, Rietveld F, Remeijer L, Beekhuis W, Binder P. A new surgical technique for deep stromal, anterior lamellar keratoplasty. Br J Ophthalmol 1999; 83(3): 327-333.
21 Muller L, Kaufmann C, Bachmann LM, Tarantino-Scherrer JN, Thiel MA, Bochmann F. Changes in intraocular pressure after descemet stripping automated endothelial keratoplasty: a retrospective analysis. Cornea 2015; 34(3): 271-274.

22 Bourcier T, Thomas F, Borderie V, Chaumeil C, Laroche L. Bacterial keratitis: predisposing factors, clinical and microbiological review of 300 cases. Br J Ophthalmol 2003; 87(7): 834-838. 